\title{
Dans le sillage de La main à la pâte Des Maisons pour la science au service des professeurs
}

Pierre Léna (pierre.lena@obspm.fr)

Membre de l'Académie des sciences, Président d'honneur de la Fondation La main à la pâte

\section{En France, près de 800000}

enfants entrent chaque année

à l'école primaire et vont

cheminer jusqu'en fin

de collège. Plus d'un tiers

d'entre eux quittera le collège

en situation d'échec

ou d'orientation non choisie.

Pour eux, comme pour une

majorité de ceux qui rejoignent

le lycée général, le goût

des sciences n'aura guère

été éveillé, ou aura disparu.

Depuis 1996, l'Académie

des sciences, avec La main à

la pâte, tente d'accompagner

les professeurs pour inverser

cet état de fait. Une nouvelle

étape est en cours, avec des

Maisons pour la science,

destinées à mettre enseignants

de primaire et de collège au

sein des universités, en lien

avec la science et la technique

vivantes, de façon structurée.
Offrir à chaque professeur enseignant les sciences, qu'il le fasse au collège ou à l'école primaire - scolarité obligatoire, où le cursus est identique pour tous les élèves -, un lieu permanent au sein de l'université où ce professeur se sente à l'aise, comme chez lui, pour dialoguer activement avec des acteurs de la science et de la technique, voici l'idée qui guide depuis 2012 la création progressive des neuf Maisons pour la science au service des professeurs, ainsi que leur action au quotidien [1]. En Alsace, Aquitaine, Auvergne (encadré, p. 32), Bretagne, Centre-Val de Loire, Dauphiné, Lorraine, Midi-Pyrénées (encadré, p. 33), Nord-Pas de Calais, s'ouvrent progressivement ces lieux de partage. Ils veulent être de modestes prototypes, au service d'un objectif qui s'impose, notamment au vu des résultats des élèves de France dans les enquêtes PISA [2] et des difficultés de recrutement de professeurs de collège [3] : permettre aux enseignants de tisser des liens avec une science et une technique vivantes, en participant à des actions de développement professionnel conçues et conduites en coopération entre mondes scientifique, industriel et éducatif.

L'idée vient de loin. Les profonds changements de nos sociétés imposent à chaque métier une constante évolution. Les lois sur la formation continue ont reconnu cet impératif. Pourtant, à rebours et comme le soulignent de multiples rapports, la profession enseignante a vu depuis vingt ans une progressive dégradation, sinon disparition, des actions en ce sens, malgré les plans académiques de formation des rectorats [4]. Le recrutement pour ce métier s'en ressent, l'enseignement des sciences en souffre cruellement. L'Académie des sciences, depuis 2007, a lancé aux pouvoirs publics plusieurs cris d'alarme [5]. Avec son action
La main à la pâte, issue de la géniale intuition de Georges Charpak en 1996 et soutenue avec Yves Quéré par nombre de physiciens, elle a pu mesurer depuis 1996 combien l'intérêt des jeunes élèves et la qualité de la pédagogie pouvaient changer en profondeur pour peu que les professeurs soient accompagnés solidement et mis en contact avec une science vivante [6]. Elle a vérifié également combien les actions, souvent remarquables, mises en place par des associations de professeurs (1'UdPPC (a) l'APBG $^{(\mathrm{b})}$ ou d'autres), ne touchaient qu'une petite fraction de ce public, le plus souvent en lycée. Le grand emprunt 2010 (Investissements d'avenir [7]) offrit en 2011 l'occasion de proposer une action plus vaste, ciblée sur les sciences en primaire et au collège : les Maisons pour la science au service des professeurs.

Chaque Maison, dont les moyens sont garantis sur cinq années, offre aux professeurs d'une académie donnée des actions de développement professionnel, d'une durée variable allant d'une demi-journée à deux jours, tout au long de l'année. La conception et la mise en œuvre de ces actions assurent, à parité, la présence d'acteurs de la science aux côtés de pédagogues, en lien avec les rectorats.

En début d'année scolaire, chaque Maison publie et diffuse dans tous les établissements de son académie une offre organisée autour de rubriques explicites : "vivre la science pour l'enseigner ", " s'ouvrir à d'autres disciplines " [8], " concevoir, construire, expérimenter, exploiter " [9]. Les professeurs volontaires, dont les déplacements sont pris en charge et dont les rectorats assurent, au mieux possible, le remplacement, rencontrent chercheurs et ingénieurs, se placent en situation d'investigation comme ils le demanderont plus tard à leurs élèves, 

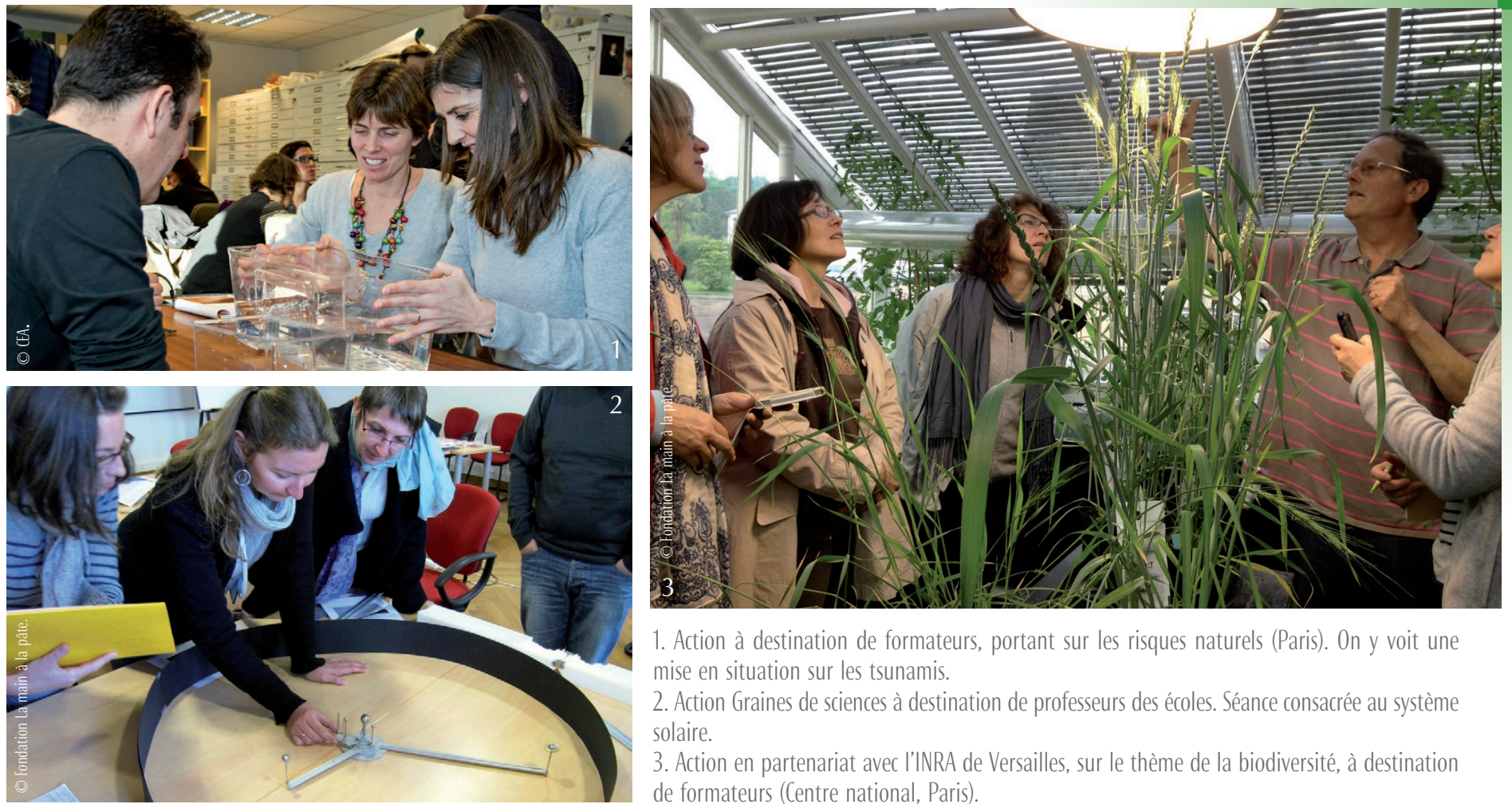

1. Action à destination de formateurs, portant sur les risques naturels (Paris). On y voit une mise en situation sur les tsunamis.

2. Action Graines de sciences à destination de professeurs des écoles. Séance consacrée au système solaire.

3. Action en partenariat avec I'INRA de Versailles, sur le thème de la biodiversité, à destination de formateurs (Centre national, Paris).

visitent laboratoires, bureaux d'étude ou entreprises, pratiquent une approche pluridisciplinaire d'une question, s'ouvrent à des champs nouveaux tels l'informatique, l'histoire des sciences ou les sciences cognitives, entreprennent éventuellement un parcours qui peut les conduire à une certification que délivrera l'université d'accueil de la Maison. En 2012-2013, ce sont près de 4600 personnes-jours qui ont vécu un rapprochement actif avec la science. Cette année, on en comptera non loin du double. Donnons quelques extraits parmi plus de 200 actions proposées cette année : Prendre la mesure (Midi-Pyrénées et IREM) ; Le hasard ne sourit qu'aux esprits bien préparés (Alsace) ; Nanotechnologies : petite échelle et grand essor (Lorraine); Le temps et ses marques (Auvergne); Sciences $d u$ numérique : langage et algorithmes (Centre national, INRIA).

Pour mettre en œuvre ce projet ambitieux, l'Académie des sciences et les deux Écoles normales supérieures (Paris et Lyon) ont proposé une Fondation de coopération scientifique, la Fondation La main à la pâte, créée par décret en 2011 et financée par les cofondateurs, les ministères, des entreprises partenaires $^{(c)}$ et naturellement les Investissements d'avenir. La coordination du réseau des Maisons, l'animation des échanges et expériences sont assurées par une équipe permanente d'une trentaine de personnes, travaillant à Paris au sein de cette Fondation dirigée par David Jasmin et Béatrice Salviat [10]. En outre, la
Fondation a repris les activités plus «traditionnelles " de La main à la pâte, tel le site Internet qui, avec 300000 visites mensuelles depuis sept ans, offre une large palette de ressources pour la classe, ou l'action internationale qui chaque année rassemble à Paris, lors d'un séminaire, plusieurs dizaines de pays hors Europe.

Désormais financées jusque 2018 - à l'exception des Maisons d'Alsace et de Midi-Pyrénées, mieux dotées au départ -, ces Maisons doivent d'abord séduire les enseignants par la qualité de leur offre, mais aussi positionner leur action vis-à-vis des toutes nouvelles Écoles supérieures du professorat et de l'éducation $(\mathrm{ESPE})^{(\mathrm{d})}$. Il est espéré que cet effort spécifique voulu par l'État et ciblé sur les sciences, mais limité à cinq ans par son financement, contribuera à la rénovation de la "formation continue " dont les ESPE ont la charge, pour autant que les leçons de ces prototypes soient concluantes. D'ores et déjà, les Maisons, accompagnées de satellites qui délocalisent leur action au plus près des enseignants, manifestent une mobilisation remarquable de la communauté scientifique et une grande créativité.

(a) UdPPC : Union des professeurs de physique et de chimie.

(b) APBG : Association des professeurs de biologie et géologie.

(c) À ce jour, BioMérieux, Michelin, Saint-Gobain, Total.

(d) ESPE : Écoles supérieures du professorat et de

l'éducation, créées en 2013 pour succéder aux IUFM et former les professeurs.

\section{Références}

1• www.maisons-pour-la-science.org

2• Principaux résultats de l'Enquête PISA 2012.

Ce que les élèves de 15 ans savent et ce quills peuvent faire avec ce quiils savent. OCDE, Paris. www.oecd.org/pisa/keyfindings/pisa-2012-resultsoverview-FR.pdf .

• www.education.gouv.fr/cid73088/donnees statistiques-des-concours-capes-session-2013.html

4- Retenons parmi ces nombreux rapports : La formation initiale et continue des maitres, Rapport conjoint des Inspections générales de l'éducation nationale (février 2003).

www.education.gouv.fr/cid2120/la-formationinitiale-et-continue-des-maitres.html

5• En particulier : La formation continue des professeurs enseignant les sciences à lécole, au collège, au lycée, Avis de l'Académie des sciences (30 novembre 2010). www.academie-sciences.fr/activite/enseign/ ressource.htm

6• Voir notamment G. Charpak, P. Léna, Y. Quéré, L'Enfant et la science, 0. Jacob (2005).

P. Léna, Enseigner c'est espérer, Le Pommier (2012). A. Bentolila et Y. Quéré, Langue et science, Plon (2013).

7• A. Juppé, M. Rocard, Investir pour l'avenir, rapport remis au président de la République (2010). http://investissement-avenir.gouvernement.fr/sites/ default/files/user/Rapport\%20\%20JUPPE\%20 ROCARD.pdf .

8 Voir La mise en cuvre du sode commun de connaissances et de compétences au collège. Rapport d'information de la commission des affaires sociales et de l'éducation, №2446 (avril 2010).

9• L'ensemble des offres est disponible sur le site www.maisons-pour-la-science.org

10• www.fondation-lamap.org

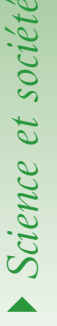




\section{La Maison pour la science en Auvergne}

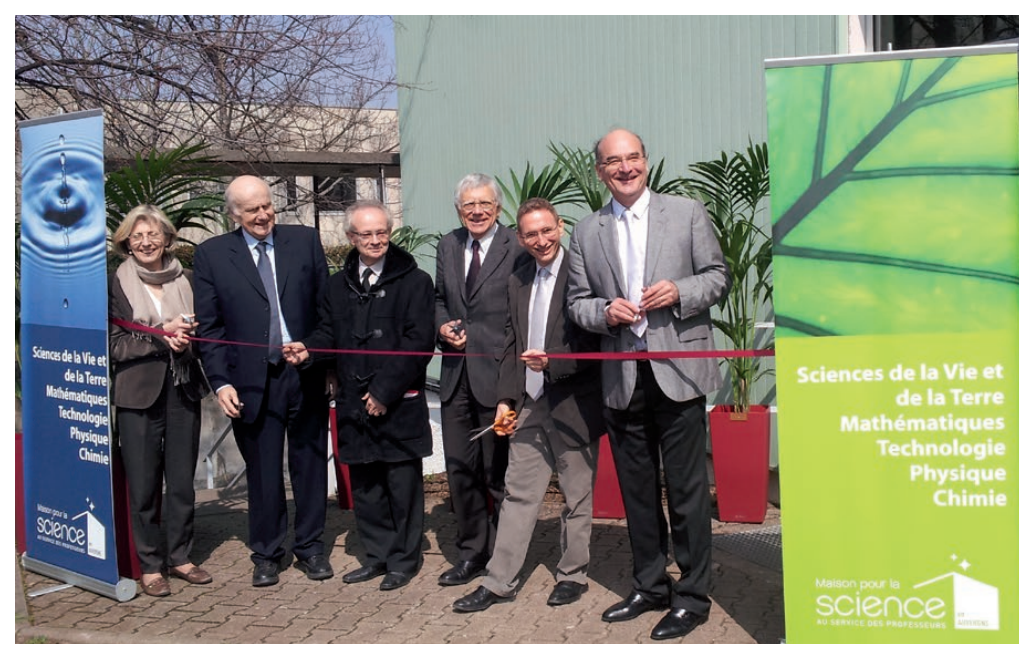

E1a. Inauguration de la Maison pour la science en Auvergne, le 27 mars 2013

De gauche à droite : Marie-Danièle Campion, recteur de l'académie de Clermont-Ferrand, Jean-François Bach, secrétaire perpétuel de l'Académie des sciences, Alain Bouvier, ancien recteur de l'académie de Clermont-Ferrand, Pierre Léna, Mathias Bernard, président de l'université Blaise Pascal, et Jean-François Mathiot.

\section{Marie Curie et la radioactivité en Auvergne}

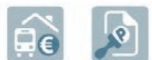

Lieu : Centre régional
Dates : Octobre 2012
Durée: 2 jours

Action idéale pour :

Professeurs du premier degré (cycle 3) et du collège

Présentation de l'action :

A l'occasion d'une exposition à la Bibliothèque Clermont Université, les multiples facettes de la vie et de l'œuvre scientifique de Marie Curie sont abordées : la découverte de la radioactivité, l'enseignement des sciences à l'école, la place des radioactivité, l'enseignement des sciences à l'école, la place des
femmes dans le domaine de la science, I'héritage scientifique de Marie Curie.

\section{Ce que les participants feront :}

- Replacer les découvertes scientifiques de Marie Curie dans une perspective historique ;

- mettre en situation quelques exemples des « Leçons de Marie Curie » dans le cadre d'un enseignement fondé sur l'investigation:

- se familiariser avec la mesure du radon en Auvergne.

Fonctions des intervenants :

Fonctions des intervenants :
Chercheurs, formateurs du premier et du second degrés

Partenaires:

Bibliothèque Clermont Université

Laboratoires de recherche
Depuis la rentrée scolaire 2012, la Maison pour la science en Auvergne propose à l'ensemble des enseignants en école primaire et en collège des formations ancrées profondément dans la science vivante. Elle le fait d'abord par le choix des thèmes proposés, mais aussi par la façon de construire et d'animer ses formations : formateurs du premier degré, du second degré, chercheurs et enseignants-chercheurs travaillent ensemble afin de permettre aux enseignants d'aborder les sciences de façon particulièrement riche et motivante.

Au vu des différentes enquêtes de satisfaction qui sont faites à l'issue de chaque formation, ce pari de l'Académie des sciences est d'ores et déjà largement réussi ! L'éventail des formations (dont un exemple est donné sur la figure E1b) proposées par la Maison pour la science en Auvergne [11] permet tout à la fois d'aborder de façon pratique des sujets scientifiques essentiels ("comprendre la radioactivité ») même s'ils ne sont pas au programme de l'école primaire ou du collège -, de pratiquer l'enseignement des sciences fondé sur l'investigation en le replaçant dans une perspective historique motivante ("les leçons de Marie Curie et la démarche d'investigation en sciences »), ou encore d'aborder les mathématiques et la science informatique de façon nouvelle et originale ("faire de l'informatique à l'école sans ordinateur »).

La Maison pour la science en Auvergne, par sa structure relativement légère et son indépendance financière, permet de surmonter un certain nombre de barrières : celle entre école primaire et collège tout d'abord, pour que le socle commun de connaissances, de compétences et de culture prenne tout son sens ; celle entre disciplines pour montrer, au-delà des spécificités de chacune, I'universalité de la Science et du raisonnement scientifique; celle enfin qui parfois subsiste entre formateurs des premier et second degrés d'une part et chercheurs et enseignant-chercheurs d'autre part (avec leurs différentes tutelles). Les enseignants peuvent ainsi profiter au mieux de la réflexion scientifique qui se développe au sein des laboratoires de recherche, et de toutes les avancées scientifiques et technologiques modernes, tout en travaillant sur les pratiques pédagogiques qu'ils pourraient mettre en œuvre dans leur classe, et se les approprier.

Ce programme est particulièrement ambitieux. II est à la hauteur des enjeux de société majeurs qui se posent actuellement pour l'enseignement des sciences à l'école, comme le rappelle Pierre Léna. La Maison pour la science en Auvergne, en collaboration étroite avec I'IREM(e) de Clermont-Ferrand et tout comme les autres Maisons pour la science, $y$ travaille avec beaucoup de passion.

Jean-François Mathiot

(jean-francois.mathiot@maisons-pour-la-science.org) Laboratoire de physique corpusculaire, Clermont-Ferrand Directeur de la Maison pour la science en Auvergne

(e) IREM : Institut de recherche sur l'enseignement des mathématiques. [11] www.maisons-pour-la-science.org/fr/auvergne 


\section{La Maison pour la science en Midi-Pyrénées}

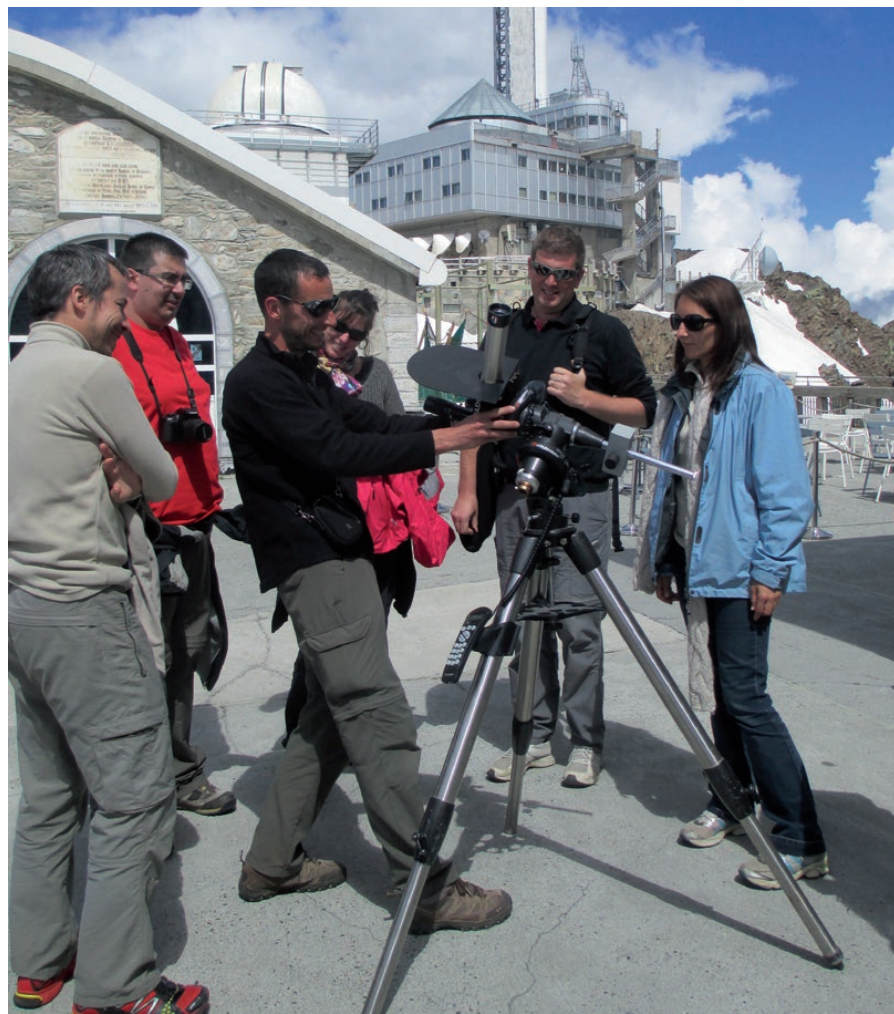

E2a. Stage de trois jours au Pic du Midi

\section{Témoignage}

La Maison pour la science au service des professeurs est devenue un partenaire clé de la formation continue professionnalisante au sein de l'Université de Toulouse. Elle a été pensée dans la logique de "formation tout au long de la vie " et de "parcours individualisés " des enseignants, incluant une cohérence entre formation initiale, "de début de carrière ", et continue, tout ceci avec cultures scientifique et technologique au cour $d u$ dispositif. Elle est une réponse au constat que l'échec scolaire se creuse en France, un des leviers étant une meilleure formation des enseignants par l'actualisation de leurs savoirs disciplinaires et l'apprentissage de nouveaux savoirs favorisant l'interdisciplinarité, par l'innovation pédagogique et technologique, ainsi que par la réponse aux besoins de terrain.

Nous avons construit notre Maison comme une interface entre mondes de la recherche - impliquant acteurs universitaires et chercheurs et de la formation des enseignants; proposer des formations mobilisant les résultats de la recherche nous a semblé pouvoir favoriser l'innovation pédagogique en prise avec l'évolution des programmes.

Dernier fait saillant : nous avons également tenu à ce que ce dispositif ait des antennes hors capitale régionale, de manière à favoriser le déploiement d'actions de formation au plus près des lieux d'exercice des enseignants, en collaboration étroite avec d'autres acteurs tels l'École supérieure du professorat et de l'éducation (ESPE) ou le très prochain Institut de Recherche sur l'Enseignement des Sciences (IRES).

Arnaud Le Padellec Maître de conférences à I'Université Paul Sabatier - Toulouse III
La Maison pour la science en Midi-Pyrénées est un lieu de rencontre, de rapprochement et d'échanges entre deux mondes, celui de l'éducation et celui de la science.

Sa principale mission est de proposer une vision actuelle et renouvelée des sciences et de leur enseignement et d'aider les professeurs d'école primaire et ceux du collège concernés par l'enseignement des sciences et de la technologie, à tisser ou renforcer des liens avec la science en train de se construire, en leur proposant de nouvelles modalités de développement professionnel, centrées sur les sciences de la vie et de la Terre et les sciences physiques et chimiques, mais également sur I'ingénierie, les sciences cognitives et les mathématiques.

Elle est pilotée au niveau national par la Fondation La main à la pâte, et, localement, par la Communauté d'universités et d'établissements "Université de Toulouse », en partenariat avec le rectorat de l'académie de Toulouse, I'Université Paul Sabatier et I'Université du Mirail.

Pour couvrir au mieux le territoire académique, la Maison pour la science en Midi-Pyrénées est implantée sur différents sites : un centre régional situé dans la halle technologique sur le campus scientifique de Rangueil à Toulouse, et des centres satellites (de Cahors, de Fleurance, du Tarn, et trois autres centres satellites en cours de création).

Depuis près d'un an, l'équipe de la Maison pour la science en MidiPyrénées, qui se compose de formateurs et de scientifiques, propose aux enseignants d'école et de collège de toute l'académie des actions de formation autour de l'astronomie, des ressources géologiques locales, des nanotechnologies, de l'immunologie, la mesure, les couleurs... ainsi que des stages en immersion de plusieurs jours au Pic du Midi (fig. E2a), dans un laboratoire de mathématiques, au centre pédagogique sur le développement durable de Fleurance, ou à la station d'écologie expérimentale du CNRS à Moulis.

Quelques chiffres

Pour la première année 2012-2013 : 56 actions menées, 1750 participants, 55 scientifiques impliqués, et 266 étudiants (dont 190 de l'Université Paul Sabatier) engagés dans le dispositif ASTEP ${ }^{(f)}$.

Pour 2013-2014 : plus de 110 actions, pour environ 4000 participants, une centaine de scientifiques impliqués, et plus de 300 étudiants participant à I'ASTEP.

Clémentine Transetti (clementine.transetti@univ-toulouse.fr) Directrice de la Maison pour la science en Midi-Pyrénées

(f) ASTEP : «Accompagnement en science et technologie à l'école primaire ", qui se propose de favoriser l'engagement des chercheurs, ingénieurs, techniciens d'entreprise et étudiants de formation scientifique au bénéfice des enseignants de l'école primaire et de leurs élèves (www.fondation-lamap.org/fr/astep ).

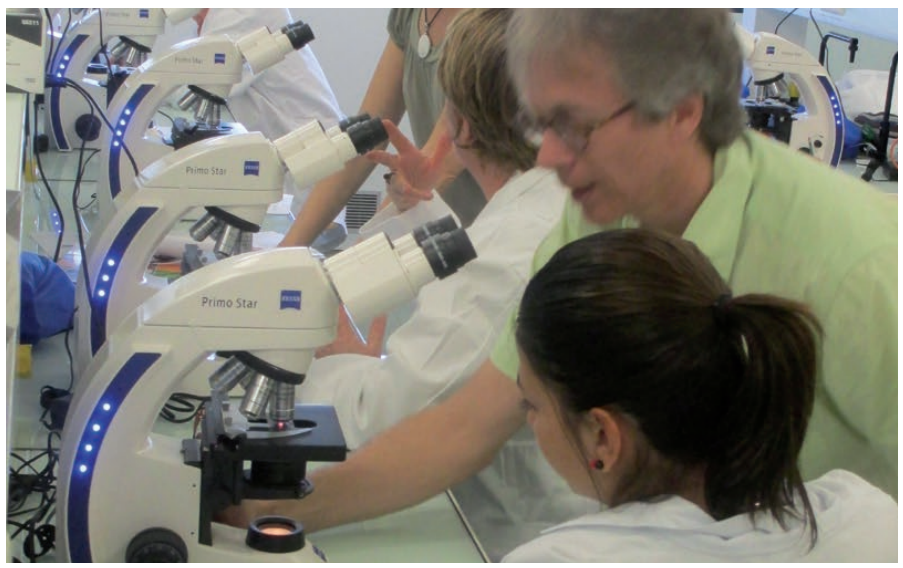

E2b. Formation en biologie cellulaire 